\title{
Buenas prácticas en la atención prenatal a adolescentes embarazadas: perspectivas de profesionales de la salud
}

\section{Good prenatal care practices in teenage pregnancy from the perspective of healthcare professionals}

\section{Boas práticas no atendiumento pré-natal para adoles- centes grávidas: perspectivas de profissionais da saúdle}

Odette del Risco-Sánchez, $\mathrm{MSc}^{1}$; Erika Zambrano-Tanaka, $\mathrm{PhD}^{2}$; Natividad GuerreroBorrego, $\mathrm{PhD}^{3}$; Fernanda Surita, $\mathrm{MD}, \mathrm{PhD}^{4}$

Recibido: 27 de abril del 2021/Aceptado: 5 de agosto del 2021

\section{RESUMEN}

Objetivo: sistematizar buenas prácticas de atención prenatal en un servicio de referencia para adolescentes embarazadas en Campinas, São Paulo, Brasil, desde la perspectiva de profesionales de la salud.

Materiales y métodos: estudio cualitativo desarrollado entre junio y julio de 2017, se emplearon entrevistas semiestructuradas y se realizó un grupo focal con 10 profesionales de la salud que prestaban atención a adolescentes embarazadas en una unidad hospitalaria del sistema público de la región de Campinas, São Paulo, Brasil. Los datos fueron examinados mediante la técnica de análisis de contenido temático de donde surgieron nuevas categorías de análisis. Se hizo validación a través de triangulación de los resultados. Se identificó la aplicabilidad teórica y práctica de los principales hallazgos.

* Correspondencia: Odette del Risco Sánchez. Departamento de Tocoginecologia. Faculdade de Ciências Médicas. Universidade Estadual de Campinas. Rua Alexander Fleming, 101, CEP:13083-881, Campinas, SP, Brasil. E-mail: oderisco89@gmail.com

1. Psicóloga. Doutoranda do Programa de Pós-graduação em Tocoginecologia Faculdade de Ciências Médicas. Universidade Estadual de Campinas (UNICAMP), Campinas (Brasil).

2. Enfermeira Obstétrica. Docente da Faculdade de Enfermagem da Universidade Estadual de Campinas, Área da Saúde da Mulher e do Recém Nascido (UNICAMP), Campinas (Brasil).

3. Psicóloga. Especialista del Centro Nacional de Educación Sexual (CENESEX), Departamento de Investigación y Docencia, La Habana (Cuba).

4. Médica. Professora Associada da Faculdade de Ciências Médicas. Universidade Estadual de Campinas (UNICAMP), Campinas (Brasil).
Resultados: fueron elaboradas tres categorías emergentes del discurso de los profesionales y de las evidencias en la literatura: 1) análisis integral de las adolescentes en situación de embarazo; 2) experiencias profesionales en la atención a las adolescentes embarazadas que evidencian calidad en el servicio; 3) fortalezas y oportunidades del servicio con posibilidades de replicación como modelo asistencial. Son consideradas buenas prácticas durante el cuidado prenatal aquellas que priorizan la atención integral a las adolescentes embarazadas; estas son coordinadas por un equipo multidisciplinario, y consiguen tener un abordaje multidimensional, sobre todo, valorando la complejidad que supone la maternidad en la adolescencia.

Conclusión: dentro de los servicios de salud sexual y reproductiva es recomendable el desarrollo de estrategias educativas, el fortalecimiento de la atención en grupos y la inclusión de la red de apoyo de las adolescentes en la atención, con el fin de favorecer una experiencia positiva durante el cuidado prenatal cuando el proceso de volverse madre acontece durante la adolescencia.

Palabras clave: atención prenatal, servicios de salud del adolescente, salud reproductiva, personal de salud, embarazo en adolescencia, salud de la mujer. 


\section{ABSTRACT}

Objective: To systematize good prenatal care practices in a referral center for pregnant teenagers in Campinas, São Paulo, Brazil, from the perspective of healthcare professionals.

Material and methods: Qualitative study conducted between June and July 2017 using semi-structured interviews and a focus group with 10 healthcare professionals providing care to pregnant teenagers in a public hospital in the region of Campinas, São Paulo, Brazil. Data were examined using the thematic content analysis technique, which gave rise to new analytical categories. Triangulation of the results was used for validation. The theoretical and practical applicability of the main findings was identified.

Results: The study looked into three categories arising from the discourse of the professionals and the evidence found in the literature: 1) comprehensive analysis of pregnant teenagers; 2) professional experiences related to the care of pregnant teenagers reflecting service quality; and 3) strengths and improvement opportunities potentially replicable as a care model. Good practices in prenatal care are those which prioritize comprehensive care for pregnant teenagers, are coordinated by a multidisciplinary team and result in a multidimensional approach, valuing the complexity of motherhood in the teenage years.

Conclusion: It is advisable to develop education strategies, strengthen group care and involve the teenager's support network as part of sexual and reproductive health services in order to promote a positive experience during prenatal care when the process of becoming a mother takes place during adolescence.

Keywords: Prenatal care, adolescent health services, reproductive health, health care providers, pregnancy in adolescence, women's health.

\section{RESUMO}

Objetivo Sistematizar as boas práticas de atenção prénatal em um serviço de referência para adolescentes grávidas em Campinas, São Paulo/Brasil desde a perspectiva dos professionais da saúde.
Materiais e métodos: Estudo qualitativo desenvolvido entre junho e julho de 2017. Empregaram-se entrevistas semiestruturadas e grupo focal com 10 profissionais da saúde que prestavam atendimento à adolescentes grávidas em uma unidade hospitalar da rede pública da região de Campinas, São Paulo/Brasil. Os dados foram analisados por meio da técnica de análise de conteúdo temática onde surgiram as categorias de análise. Realizou-se validação através de triangulação dos resultados e foi identificada a aplicabilidade teórica e prática dos principais achados.

Resultados: Foram elaboradas três categorias emergentes do discurso dos professionais e das evidências na literatura: 1) análise compreensiva das adolescentes em situação de gravidez; 2) vivências profissionais no cuidado de adolescentes grávidas que mostram a qualidade no atendimento; 3) potencialidades e oportunidades do serviço com possibilidades de replicação como modelo assistencial. No atendimento pré-natal, são consideradas como boas práticas aquelas que priorizam a atenção integral à gestante adolescente; as que são coordenadas por uma equipe multidisciplinar e conseguem ter uma abordagem multidimensional, sobretudo, avaliando a complexidade que supõe a maternidade durante a adolescência.

Conclusões: Dentro dos serviços de saúde sexual e reprodutiva, é recomendável o desenvolvimento de estratégias educativas, o fortalecimento da atenção em grupos e a inclusão da rede de apoio das adolescentes na atenção para favorecer uma experiência positiva durante o cuidado pré-natal levando em consideração o processo de tornar-se mãe durante a adolescência.

Palavras-chave: cuidado pré-natal, serviços de saúde do adolescente, saúde reprodutiva, pessoal de saúde, gravidez na adolescência, saúde da mulher.

\section{INTRODUCCIÓN}

La adolescencia puede ser comprendida como el periodo del desarrollo donde acontecen transformaciones biopsicosociales que favorecen la preparación para asumir roles propios de la adultez (1). Pinto y cols mencionan que la Organización Mundial de la Salud (OMS) 
ha definido como adolescentes a aquellas personas en edades comprendidas entre 10 y 19 años, reconociendo que las características individuales y los elementos socioculturales deben ser tomados en consideración para comprender este periodo etario (2).

En América Latina y el Caribe (ALC) los y las adolescentes representan el 18\% del total de la población (3). En Brasil se estima que 15\% de la población se encuentra entre los 10 y 19 años (4). Si bien los adolescentes han estado presentes en las agendas públicas, ello no ha sido suficiente para reducir el impacto de las desigualdades socioeconómicas y las limitaciones para el acceso a servicios de educación y salud de calidad que afectan a este grupo poblacional, como por ejemplo en temas asociados a su salud sexual y reproductiva, y en especial a la prevención del embarazo en la adolescencia.

La OMS estima que anualmente alrededor de 16 millones de adolescentes se vuelven madres, la mayoría vive en países de bajos y medianos ingresos (5). ALC es considerada la región con la segunda tasa de embarazo adolescente más alta (6). La Organización Panamericana de la Salud ha identificado que el 38\% de las mujeres en la región tienen un embarazo antes de cumplir 20 años (7). En Brasil, durante el periodo 2010-2018 la tasa de natalidad fue de 52,7 por cada mil adolescentes entre 15 y 19 años (8). Entre los factores asociados al embarazo adolescente en la región se encuentran: la violencia y el abuso sexual, los valores culturales y las normas de género que perpetúan relaciones desiguales, la transmisión intergeneracional de la pobreza, las dificultades para el acceso a servicios de salud sexual y reproductiva, sobre todo a métodos contraceptivos, así como deficiencias y falta de programas destinados a la educación integral de la sexualidad (6). En este sentido, la gestación en edades tempranas puede acarrear consecuencias para el bienestar físico y psicosocial de la adolescente (2, 9-13). Entre las complicaciones clínicas más recurrentes se encuentran la preeclampsia, anemia, prematuridad y bajo peso al nacer, y desde el punto de vista social posiciona a las adolescentes en situación de vulnerabilidad, ya que limita las oportunidades de empleo y la obtención de ingresos dignos, aumenta los riesgos de evasión escolar e incide en la reproducción de la pobreza y la exclusión social (5).

Con el propósito de contribuir a la reducción del embarazo en la adolescencia han sido adoptadas varias medidas que incluyen el diseño de planes y estrategias regionales, subregionales y nacionales; no obstante, ello no ha sido suficiente para lograr que cada gestación sea intencional, planificada y concebida en edades que no interrumpan el desarrollo psicosocial de las adolescentes (6). Como respuesta a la gestación en estas edades, los servicios de salud han diseñado estrategias de cuidado entre las que se encuentran la atención humanizada y de calidad durante el embarazo y el puerperio para disminuir posibles complicaciones clínicas, tanto maternas como neonatales (13). Los profesionales de la salud son clave para la atención integral a las adolescentes, sobre todo en el desarrollo de estrategias diferenciadas para este grupo etario (14). Sin embargo, resulta necesario transitar de modelos asistenciales hegemónicos (15) hacia una comprensión integral de la maternidad en la adolescencia.

En coherencia con este propósito, productos de transferencia del conocimiento como las Recomendaciones de la OMS sobre Atención Prenatal para una experiencia positiva del embarazo (16), son referentes para desarrollar servicios de atención prenatal que posicionen a mujeres y adolescentes en el centro de atención, proveyéndoles la atención de calidad que desean y necesitan. En Brasil, recursos como la Rede Cegonha (17) y el Programa de Humanização do Pré-Natal e Nascimento (18) establecen protocolos para garantizar atención humanizada y segura a mujeres gestantes y puérperas. De igual modo, las evidencias aportadas por la OMS sobre la atención prenatal reconocen que los contactos entre las gestantes con los servicios prenatales ofrecen mayor seguridad e influyen en una experiencia positiva durante el embarazo, parto y puerperio (16). Sin embargo, los análisis en cuanto a la asistencia prenatal requieren ser discutidos no solo desde una perspectiva cuantitativa, sino también cualitativa que permita la evaluación de la calidad del contacto entre profesionales de salud y adolescentes en estos servicios. 
Existen varios instrumentos que guían los cuidados deseables para la atención prenatal (16) y el diseño de servicios de salud de calidad para adolescentes (19), uno de los posibles modelos contempla la evaluación de diferentes dimensiones, tales como calidad científicotécnica, accesibilidad, atención centrada en el paciente y seguridad (20). Si bien los marcos de referencia que cubren el concepto de la calidad de los servicios en salud pueden ser estudiados desde diversos modelos y aristas, tanto de usuarios, líderes, personal de salud como de formuladores de políticas (21), investigaciones sobre los servicios de atención prenatal han señalado la carencia de datos sobre las percepciones de los profesionales de salud en torno a su quehacer, resaltando la importancia de rescatar la subjetividad de estos profesionales para realizar ajustes a las políticas de salud (22). Por lo tanto, resulta relevante explorar las percepciones sobre las prácticas de salud destinadas a adolescentes embarazadas, desde las experiencias de equipos multiprofesionales, con miras a su aplicación y adaptación a otros contextos en la región. De igual modo, realizar una evaluación cualitativa desde el discurso de los proveedores sobre la atención prenatal a adolescentes contribuye a la reflexión en torno a sus experiencias y a la identificación de estrategias para mejorar sus prácticas.

De esta manera, en este estudio la investigación tiene como objetivo sistematizar buenas prácticas de atención prenatal en un servicio de referencia para adolescentes embarazadas en Campinas, Brasil, desde la perspectiva de profesionales de la salud.

\section{MATERIALES Y MÉTODOS}

Diseño y población. Se realizó un estudio cualitativo con el propósito de comprender en profundidad las percepciones de proveedores de salud sobre su práctica profesional, empleándose como procedimiento metodológico el análisis de contenido temático para la realización de inferencias sobre el objeto de estudio. Considerando la naturaleza del objeto, fue definido este abordaje metodológico, pues en el campo de la salud sexual y reproductiva, las investigaciones cualitativas resultan relevantes en la comprensión de los significados atribuidos a los comportamientos que permean las prácticas de salud (23).

Con este propósito, fue seleccionada una unidad hospitalaria de la región de Campinas, São Paulo, con servicio especializado para la atención prenatal a adolescentes (PNA). El Hospital Prof. José Aristodemo Pinotti-Centro de Atenção Integral à Saúde da Mulher (CAISM), Universidade Estadual de Campinas (UNICAMP), posee más de 30 años de creación, hospital universitario de carácter público, centro de referencia, investigación y asistencia especializada a la salud de la mujer y del recién nacido que ofrece servicios a aproximadamente cinco millones de personas de 42 municipios del Estado, además de usuarios de otras regiones que buscan la asistencia de la institución (24). Su pertenencia al Sistema Único de Saúde (SUS), propicia la integración y articulación con el resto de la red socio-asistencial existente en la región.

El muestreo fue intencional; mediante la técnica bola de nieve se incluyeron profesionales del equipo multidisciplinario que laboraban en el PNA. Dentro del servicio estudiado fueron identificados participantes claves, quienes indicaban profesionales que pudieran ampliar la información sobre el servicio objeto de estudio, empleándose como estrategia el muestreo en cadena o por redes (25). Para evitar posibles sesgos de selección, la estrategia de muestreo contempló entre los criterios de inclusión profesionales de diferentes especialidades y que el profesional ofreciera atención especializada en el periodo de desarrollo de la investigación, con la finalidad de integrar diversas perspectivas en los análisis. Referente al tamaño de la muestra, todos los profesionales que trabajaban en el servicio durante el periodo de realización de la investigación, exceptuando dos autoras de la investigación, fueron invitados y aceptaron participar; previo consentimiento informado.

Procedimiento. Los participantes fueron contactados de forma presencial por la investigadora, quien condujo posteriormente las entrevistas, dentro de los locales donde se ofrece el servicio de atención prenatal. Los datos fueron recogidos mediante entrevistas individuales y un grupo focal. Se realizaron entrevistas semies- 
tructuradas a cada participante, las cuales tuvieron una duración media de 32,5 minutos. Las entrevistas permitieron profundizar al equipo de investigación en las experiencias profesionales de los participantes. Posteriormente, se realizó un grupo focal con cinco especialistas del equipo multidisciplinar. Este ejercicio tuvo una duración de 72 minutos, contando con una coordinadora y una co-coordinadora, quien asumió roles de observadora y relatora, ambas psicólogas con experiencia para el trabajo con grupos. Con el consentimiento de los participantes, las entrevistas fueron registradas en audio para su posterior transcripción y análisis.

Se indagó por: a) años de experiencia y profesión; b) los factores que inciden en el comportamiento de la maternidad adolescente en Brasil y en la población específica que frecuenta el servicio; c) las fortalezas, debilidades, amenazas y oportunidades del equipo multidisciplinario para la atención a la salud sexual y reproductiva de adolescentes embarazadas; d) la relación entre la atención prenatal a las adolescentes y el respeto a sus derechos sexuales y reproductivos; además de e) las competencias necesarias que debe poseer un profesional para laborar en el servicio.

Como parte de la investigación, se llevó a cabo una etapa de aculturación con la inmersión de las investigadoras en el campo, mediante la participación en las actividades habituales del servicio, lo que permitió identificar prácticas usuales en el servicio de salud. Consecutivamente, se aplicaron las técnicas de recolección y procesamiento de datos. Los datos fueron examinados mediante la técnica de análisis de contenido temático propuesta por Bardin (26) (Figura 1). Para ello, los textos fueron leídos cuidadosamente y de manera independiente por dos investigadoras para su familiarización inicial con el material y con el contexto en el cual fue producido, aprehendiendo las ideas principales y sus significados generales (27). Producto de la lectura reflexiva, fueron identificadas las unidades de análisis, las temáticas más recurrentes y relevantes, se generaron códigos, y se elaboraron las categorías, las cuales fueron refinadas en un proceso de discusión entre pares. El software NVivo versión
11 (QSR International MA, USA) auxilió al equipo de investigación en la identificación de la frecuencia de palabras y el conteo de códigos, facilitando la elaboración de categorías temáticas. Este programa contribuyó a la organización del material, codificación, elaboración de representaciones gráficas para las relaciones entre temas emergentes y categorías, así como en la validación de los análisis realizados por el equipo de investigación para la definición de las categorías. Con el fin de garantizar la confiabilidad de los datos, las categorías elaboradas fueron discutidas y validadas entre el equipo de investigación y el grupo de pesquisa Saúde Reprodutiva e Hábitos Saudáveis (SARHAS-UNICAMP).

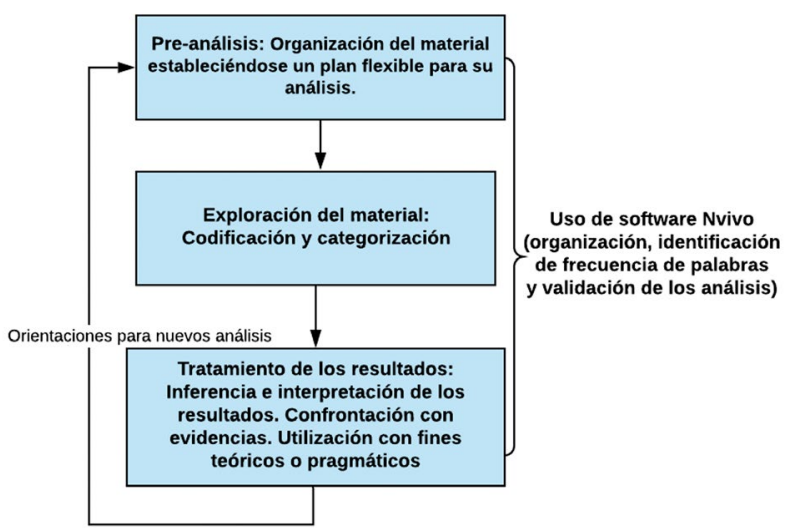

Figura 1. Representación simplificada de las fases del análisis de contenido propuesto por Bardin (2011) aplicadas en la investigación. Campinas, São Paulo, Brasil, 2017.

Fuente: elaboración propia.

El rigor metodológico de la investigación se basó en los criterios de dependencia, credibilidad y confirmación (25). La validación interna fue establecida mediante el planeamiento adecuado de los métodos, procedimientos y técnicas conforme a la naturaleza y objetivos del estudio, así como a través de la triangulación de los resultados obtenidos mediante las entrevistas, grupo focal, y evidencias en la literatura sobre atención prenatal y servicios de salud para adolescentes, facilitando la comprensión profunda del fenómeno estudiado (23, 25). Finalmente, los resultados fueron interpretados y contrastados con las evidencias existentes en la literatura sobre el objeto de estudio, e identificada la aplicabilidad teórica y práctica de los principales hallazgos. 
Aspectos éticos. El proyecto fue aprobado por el Comité de Ética en Pesquisa de la Universidade Estadual de Campinas con número do CAAE: 64530116.0.00005404. Todos los participantes firmaron el término de consentimiento libre y esclarecido. Para mantener el anonimato de los profesionales, sus discursos fueron identificados con números arábigos consecutivos, así como con letras del alfabeto: P (Profesional) y GF (Grupo Focal).

\section{RESULTADOS}

La recolección de datos se realizó entre junio y julio de 2017. En la investigación participaron 10 profesionales. La media de experiencia en el servicio fue de 13,6 años (Tabla 1). La frecuencia simple de las palabras mostró que la palabra "adolescentes" fue la de mayor recurrencia en las entrevistas. Otros vocablos usados remiten al contexto social de las adolescentes, y refuerzan la importancia del equipo profesional y de la institución. En el discurso de los entrevistados emergió la importancia de abordajes individuales y grupales en la atención ofrecida a esta población (Figura 2).

\begin{tabular}{|c|c|c|}
\multicolumn{3}{c}{ Tabla 1. } \\
Características de los profesionales estudiados. \\
Campinas, São Paulo, Brasil, 2017. Fuente: ela- \\
boración propia.
\end{tabular}

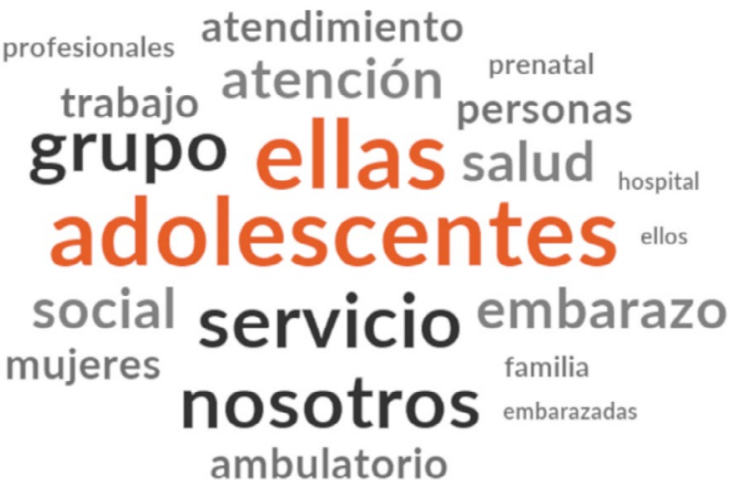

Figura 2. Nube de palabras (NVivo- QSR International MA, USA). Campinas, São Paulo, Brasil, 2017. Fuente: Elaboración propia.

Se elaboraron tres categorías temáticas emergentes de los discursos de los profesionales y de las evidencias en la literatura sobre servicios de salud para adolescentes y para la atención prenatal: a) análisis integral de las adolescentes en situación de embarazo; b) experiencias profesionales en la atención a las adolescentes embarazadas que evidencian calidad en el servicio; y c) fortalezas y oportunidades del servicio con posibilidades de replicación como modelo asistencial (Figura 3).

a) Análisis integral de las adolescentes en situación de embarazo. Los profesionales mencionaron la necesidad de lograr un abordaje diferente hacia la adolescente embarazada como principal motivo para la creación de un servicio especializado. Ellos reforzaron la valoración de las necesidades particulares de las adolescentes, debido a la confluencia de dos momentos de crisis normativas.

(...) la necesidad de un espacio diferenciado para la atención de las adolescentes embarazadas es porque ellas están en una etapa diferente de la vida. Ser adolescente, vivenciar la adolescencia, ya es un proceso complejo para algunos. En conversación con profesional 3 (junio, 2017).

(...) Emocionalmente es un momento complicado de transformaciones y búsqueda de identidad personal, social, de género. (...) la gestación es una crisis previsible dentro de un momento de crisis previsible del ciclo vital de la familia que es la adolescencia. Entonces, es la conjunción de dos momentos de crisis que desorganizan a la persona, desorganiza a la familia, desorganiza desde el punto de vista social (...). En conversación con profesional 2 (junio, 2017). 


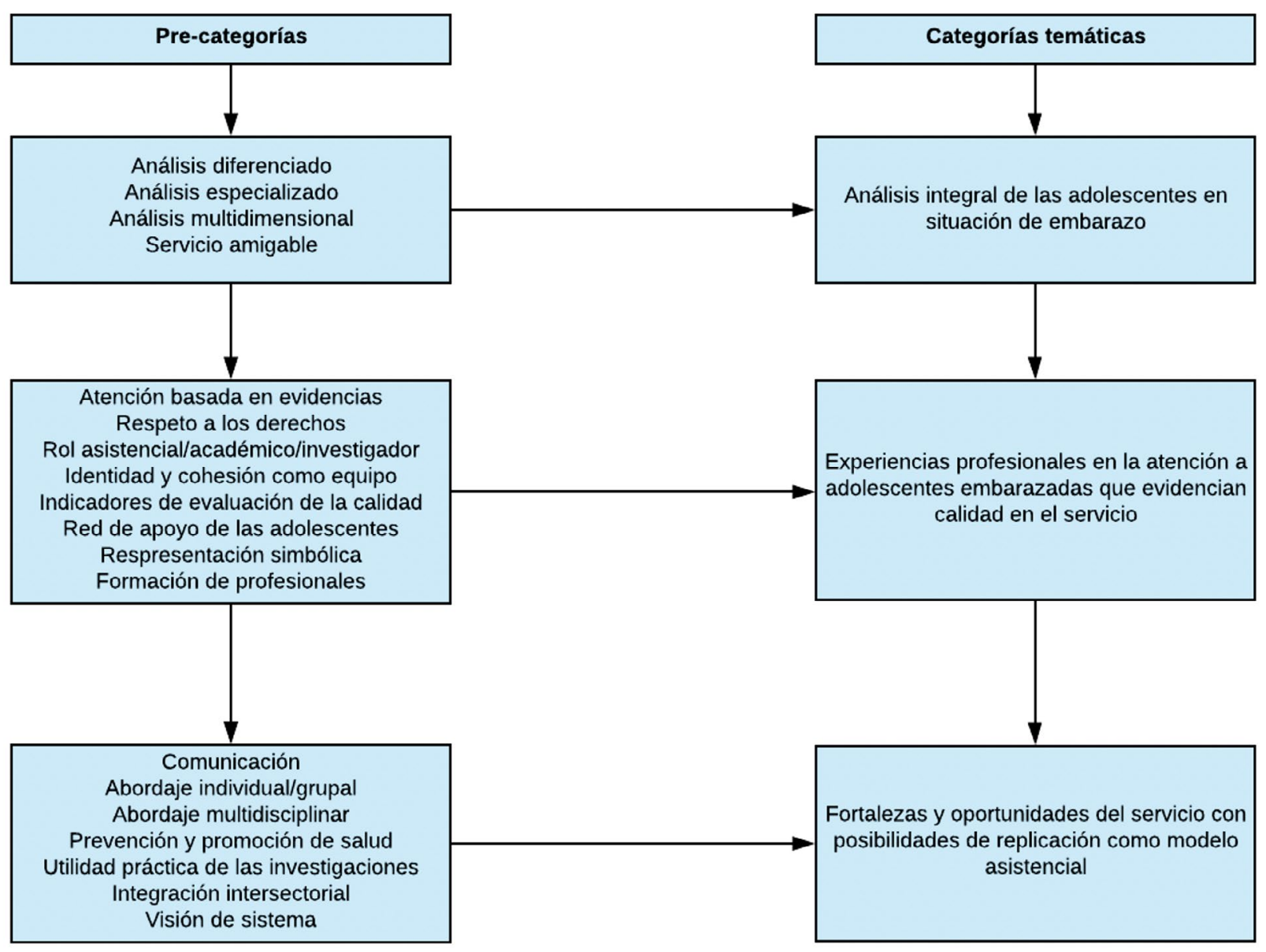

Figura 3. Flujograma de pre-categorías y categorías temáticas aplicado al estudio. Campinas, São Paulo, Brasil, 2017. Fuente: elaboración propia.

Desde el criterio de los entrevistados, la dimensión biológica no resulta la única arista a abordar, por lo que elementos socioculturales precisan ser integrados a la comprensión del embarazo en edades tempranas. En el grupo focal emergieron discursos que abordaron la hipererotización de las adolescentes por parte de los adultos, la repetición intergeneracional de la maternidad adolescente, la deserción escolar, la influencia del grupo de pares para el inicio de las relaciones sexuales, y el papel de los medios de comunicación entre los factores que inciden en la maternidad en estas edades. En este sentido, en su práctica valoran la importancia de integrar elementos psicosociales que caracterizan a las adolescentes y sus redes de apoyo, teniendo una mirada intencional al contexto familiar, escolar/laboral y emocional.
(...) yo pienso que es nuestro papel como profesional de la salud educar a esas adolescentes, hacer que ellas reflexionen (...) Yo creo que se tienen que trabajar mucho el fortalecimiento de ellas, que ellas consigan posicionarse, valorarse (...). En conversación con profesional 8 (julio, 2017).

Desde los discursos de los profesionales, se identifica la necesidad de abordar la atención a la adolescente embarazada dentro del servicio como un "todo".

Yo creo que [el servicio de atención prenatal para adolescentes] es uno de los ambulatorios más humanizados que yo percibo, porque muchas veces los otros [ambulatorios] se enfocan en la enfermedad, y este de adolescentes, no. Él se enfoca en la adolescente como un todo (...). En conversación con profesional 10 (junio, 2017). 
b) Experiencias profesionales en la atención a las adolescentes embarazadas que evidencian calidad en el servicio. Los profesionales refirieron la relevancia de los espacios grupales diseñados dentro del servicio donde participan, generalmente, dos especialistas de áreas diferentes, junto con las adolescentes que reciben atención en el servicio. Los grupos abordan temas concernientes a las principales interrogantes que las adolescentes poseen, para lo cual valoran sus interacciones e historias de vida.

(...) tenemos los grupos que hacemos mezclados, que en un momento lo hace el médico y el psicólogo, en otro lo hace el médico y la nutricionista, enfermera y asistente social y que es de libre demanda. Ellas comienzan, las presentamos y ellas traen una problemática, como ellas se están sintiendo, y aprovechamos y pasamos orientación. En conversación con profesional 1 (junio, 2017).

En el espacio grupal se abordan cuestiones que involucran desde los cuidados durante la etapa prenatal, parto y puerperio, lactancia materna, nutrición y realización de actividades físicas, hasta elementos vinculados a las dinámicas familiares y escolares, la prevención de situaciones de riesgo asociadas a la violencia, depresión, suicidio, entre otros temas que emergen según las necesidades identificadas por el equipo. Todas estas temáticas, pensadas como acciones enfocadas en el bienestar biopsicosocial de la adolescente, son desarrollados por profesionales de diferentes áreas.

Algunos de los grupos coordinados por los profesionales se realizan invitando a las adolescentes y sus respectivos acompañantes, en tanto la necesidad de valorar las redes de apoyo, ya que estas actúan como facilitadores o como barreras del proceso de cuidado y bienestar de la adolescente. Los discursos de los profesionales reflejan la importancia de las dinámicas grupales para el servicio:

(...) el grupo es el alma donde podemos pasar el máximo de informaciones para el máximo de personas posibles. En conversación con equipo multidisciplinar durante grupo focal (julio, 2017).

Los entrevistados declaran que desarrollan actividades educativas con temáticas que tienen impacto en los vínculos interpersonales de las adolescentes con sus familiares y parejas, en los desafíos que enfrentan en contextos escolares y comunitarios; incluyendo acciones que promueven el desarrollo de hábitos de vida saludables.

(...) intentamos trabajar aspectos de promoción de salud y autonomía, y empoderamiento de ellas para tener un autocuidado adecuado (...) Nosotros intentamos ofrecer autonomía para escoger en cuanto al [método de] planificación familiar. Trabajamos aspectos relacionados con la comunicación interpersonal, comunicación con los familiares (...) Además de trabajar aspectos de asertividad, comunicación, expresión de sentimientos (...) trabajar con promoción de salud mental (...). En conversación con profesional 2 (junio, 2017).

La preservación y garantía de derechos de las adolescentes fue considerado como básico, reflejando en los discursos el compromiso social que poseen los profesionales en la defensa de los derechos de quienes pueden encontrarse en una situación de vulnerabilidad.

(...) yo voy a defender los derechos de los que están en condiciones de vulnerabilidad, y también de los que sus derechos sean violados, y empleo lo que existe en la legislación para garantizar que esos derechos sean respetados. En conversación con profesional 3 (junio, 2017).

Los profesionales de esta unidad asumen un doble rol, debido a su actuación en el ámbito académico y asistencial. La existencia de grupos de pesquisa permite perfeccionar la práctica profesional, sustentada en las investigaciones que acontecen en el propio servicio. La formación de jóvenes profesionales es un recurso empleado por la institución para perpetuar la filosofía del servicio.

Yo tengo profesionales formados que vienen para hacer un año de adiestramiento profesional y están conmigo, (...) es una relación de teoría y práctica, enseñanza y aprendizaje, y socialización de conocimientos (...) existe esa función social de una universidad pública de socializar ese conocimiento. Es posible esa complementación del conocimiento, ese intercambio dentro del servicio. En conversación con profesional 3 (junio, 2017). 
Desde la percepción de los profesionales de salud, entre la población adolescente que realiza su atención prenatal en el centro, se observa una reducción del número de cesáreas. No obstante, en sus discursos también colocan énfasis en la labor realizada en la atención prenatal, especialmente, para el empoderamiento de las adolescentes para la reducción de la ansiedad y temores que genera el parto, así como para asumir los desafíos de la maternidad, siendo percibido como un indicador para evidenciar la calidad de la atención.

(...) después que ese bebé nace vemos adolescentes más organizadas, más conscientes, más confiadas, más seguras. Esas adolescentes tienen todo ese apoyo durante ese prenatal, tienen un espacio para hablar sobre sus dudas, sobre sus miedos, sobre sus cuestionamientos, ellas tienen espacio para pensar sobre ese futuro (...) yo creo que el prenatal adolescente favorece eso, que ellas tengan esa madurez para enfrentar ese desafío de la maternidad. En conversación con profesional 8 (julio, 2017).

Ante la indagación en torno a la representación simbólica sobre el servicio, emergieron varias asociaciones a vivencias personales que develan el compromiso de los profesionales con la labor desarrollada para la atención prenatal a adolescentes. Las representaciones reflejan los afectos envueltos en el servicio, dado por la necesidad de cuidado que demanda la adolescente; sobre todo, cuando se percibe por parte del equipo que se precisa de un entorno acogedor, que ofrezca a las usuarias confianza y oportunidades de crecimiento personal. Son recurrentes las referencias a las potencialidades, y las modificaciones psicosociales por las que atraviesan las adolescentes, estas últimas no vistas como estigmas o determinantes, sino como condiciones modificables.

El adolescente es una semilla en la que nosotros vamos regando en un terreno muy fértil, si se ofrece una atención de calidad, probablemente va a florecer a recoger esos frutos después, pero al mismo tiempo, si no le cuidamos, tal vez no sea un árbol. En conversación con profesional 10 (junio, 2017). c) Fortalezas y oportunidades del servicio con posibilidades de replicación como modelo asistencial. La visión multidisciplinar se constituyó como una de las principales fortalezas reconocidas por los entrevistados. La posibilidad de crear un equipo profesional cohesionado y motivado es favorecida por una cultura organizacional que promueve el intercambio entre especialistas de diferentes áreas.

Mi saber no solo está conmigo, hay cosas que solo yo sé porque yo trabajo mucho aquí, pero hay otras personas que están aptas para cubrirme también. En conversación con profesional 5 (junio, 2017).

Para llevar a cabo la atención prenatal a adolescentes, revelan la necesidad del fortalecimiento de competencias profesionales que trasciendan los conocimientos y habilidades técnicas, involucrando competencias para el trabajo en equipo y el desarrollo de habilidades comunicativas.

La competencia técnica de cada una en su área es fundamental, es básico, más allá de la competencia técnica yo creo que es esa parte del apego, acá se habla de vestir la "camiseta del equipo". En conversación con equipo multidisciplinar durante grupo focal (julio, 2017).

La implementación de los resultados de investigaciones que se desarrollan en el propio servicio y que puedan mejorar la calidad de la atención fue otro elemento señalado por los profesionales. Los entrevistados apuntaron la utilidad práctica de la producción científica generada dentro de la propia institución.

Lo que percibo del equipo de prenatal de CAISM es eso, que las personas están buscando mejorar. (...) Tienen varias investigaciones en el propio espacio que atiende a adolescentes (...) todas esas investigaciones van a servir para mejorar el servicio, para apuntar donde nosotros estamos acertando más y donde acertando menos, y eso es fundamental. En conversación con profesional 3 (junio, 2017).

La investigación científica muestra conocimientos nuevos, podemos ver cosas diferentes y aplicadas a nuestra práctica, eso es importante para la salud. En conversación con profesional 4 (junio, 2017).

El vínculo con la comunidad fue señalado como relevante para lograr las metas comunes que posee 
la red socio-asistencial. Desde el discurso de los propios profesionales, fue reconocida la necesidad de trascender el espacio médico-hospitalario para integrarse con los entornos comunitarios, familiares y escolares, dada la complejidad que supone el embarazo durante la adolescencia. Esto fortalece la visión de sistema que debe prevalecer en la red diseñada para la atención a la salud de las adolescentes. Para el desarrollo de la atención prenatal se hace evidente, a través del discurso de los profesionales, la importancia de la comunicación tanto entre los profesionales de salud, así como entre el servicio y la comunidad.

Siempre es importante que las unidades que hacen acompañamiento de prenatal adolescente tengan ese vínculo con la comunidad en la que ella está inserta, y ahí discutir con los profesionales que componen la red socio-asistencial de ese espacio, y que pueden estar dando un respaldo en el acompañamiento con esa adolescente (...) EI SUS es una vía de comunicación y de encaminamiento inmensa (. . .) Si es necesario vamos más allá de los muros de la institución para garantizar los derechos de las adolescentes. En conversación con profesional 3 (junio, 2017).

\section{DISCUSIÓN}

Los resultados encontrados develan que la atención a la adolescente embarazada precisa de un abordaje multidisciplinar frente a la complejidad que supone el proceso de convertirse en madre durante esta etapa de la vida. En este sentido, varias evidencias (28-31) destacan la necesidad de observar las peculiaridades psicosociales que permean el embarazo durante la adolescencia. En el diseño de los servicios destinados a las adolescentes, tener una visión integral favorece que los espacios disponibles respondan a las demandas específicas de esta población. Ello es relevante en la concepción de servicios amigables para adolescentes (32), lo cual resulta importante para quebrar el paradigma adultocéntrico que, en ocasiones, permea los servicios de salud (33).

Estudios realizados entre gestantes y puérperas adolescentes $(30,34)$ enfatizan en la necesidad de comprender la vivencia de la maternidad en este periodo desde las propias expectativas, experiencias y heterogeneidad que implica ser adolescente. Esta perspectiva posee valor práctico dentro de los servicios de salud, sobre todo para el diseño de servicios de calidad centrados en el paciente (20) y para el reconocimiento de la adolescente como sujeto de derechos.

A su vez, involucrar a la pareja y a la familia en el esquema de atención es una práctica observada y sugerida, en tanto que la red de apoyo resulta esencial para favorecer el ejercicio pleno de la maternidad/ paternidad. La participación paterna en la atención prenatal contribuye en la construcción de su rol, a veces expropiado desde el propio diseño de los sistemas de salud. De este modo, una revisión integradora en torno a las actitudes de los adolescentes frente al embarazo en la adolescencia (35) develó que aún existen vacíos en la producción de evidencias sobre las experiencias de adolescentes que se hacen padres, lo que resulta un paso importante para desarrollar intervenciones destinadas a este grupo. Otros autores también han reconocido la necesidad de visualizar al adolescente masculino dentro de los servicios de salud sexual y reproductiva (32).

En consonancia con otra investigación que aborda la atención prenatal a adolescentes (36) se demuestra como una práctica recomendable la implementación de acciones en el espacio grupal que aborden aspectos de la salud sexual y reproductiva, el autocuidado, cuidado del recién nacido, relaciones interpersonales, así como otros conocimientos significativos en la trayectoria de convertirse en madres. Esto involucra una visión amplia de la salud de la embarazada en términos de bienestar biológico, psicológico y social, ofreciendo la oportunidad de desarrollar estrategias educativas desde las cuales se integren diferentes saberes.

Los resultados confirman que la existencia de un equipo multidisciplinar es una buena práctica para ofrecer una atención de calidad y humanizada a las adolescentes $(37,38)$. Si bien se aprecia un alto grado de especialización de los profesionales, existe un espacio de interacción entre las disciplinas, que se hace evidente, sobre todo en la atención en gru- 
pos. Los mismos se desarrollan bajo la coordinación de especialistas formados en diferentes áreas, y se constituyen en espacios de diálogo, no solo con las adolescentes, sino entre ellas.

Por ello, el grupo no solo es importante como estrategia educativa, sino como oportunidad para la adolescente sentirse cuidada, apoyada y empoderada por sus pares, y por un equipo comprometido con su bienestar (36). A su vez, el trabajo en grupos dentro del servicio estudiado ofrece la oportunidad de desarrollar una labor educativa más efectiva, en tanto la posibilidad de transitar por los diversos temas abordados en estos espacios.

Cada vivencia en torno al embarazo se constituye como una experiencia única. En el afán de definir protocolos necesarios para estandarizar un servicio basado en evidencias científicas la creatividad e iniciativa de los equipos de salud no puede ser obviada. En este sentido, en cuanto a los tipos de abordajes para la atención prenatal (individual, grupal o mixto) (16), el servicio estudiado emplea un abordaje mixto. Más allá de las consultas individuales, el grupo es visto por los profesionales como una oportunidad para brindar orientaciones que son enriquecidas desde las propias inquietudes que emergen espontáneamente, y que, a su vez, posibilita el intercambio de experiencias entre pares.

Así mismo, se ha evidenciado que el vínculo que se establece entre el equipo multiprofesional y la adolescente resulta clave en las vivencias positivas durante el embarazo y el parto (34). El contacto continuo entre profesionales de la salud y las usuarias del servicio prenatal es un elemento que brinda la oportunidad de establecer un vínculo de confianza y una mejor comunicación entre profesionales, usuarias y sus redes de apoyo (16).

En síntesis, en el servicio estudiado fueron constatados elementos hallados en la literatura relativos a la evaluación de la calidad de los servicios de salud (20). Aspectos concernientes a la labor para garantizar la calidad científico-técnica en la atención fueron identificados, tanto en el empleo de las investigaciones que se producen en el propio servicio como en la preocupación por la formación de profesionales que puedan dar continuidad a la atención. A su vez, el desarrollo de la atención basada en evidencia científica contribuye a garantizar la seguridad de las usuarias en términos de favorecer un menor riesgo de exposición de las adolescentes a prácticas no recomendadas en el cuidado prenatal. De igual modo, tener a disposición un equipo multidisciplinar en un servicio de carácter público, especializado en la atención a las adolescentes, resulta un aspecto positivo para favorecer la accesibilidad de las usuarias. Otra cuestión a señalar como otro de los aspectos identificados en el discurso de los profesionales es la atención centrada en las necesidades y demandas de las usuarias, así como el reconocimiento de los diferentes factores que subyacen al proceso de convertirse en madre durante la adolescencia.

Como limitación del estudio se puede señalar la selección de un servicio de referencia para el desarrollo de la investigación, lo cual puede incidir en la generalización de los resultados obtenidos; no obstante, los hallazgos de la investigación pueden ofrecer pautas para obtener una idea general del problema estudiado y contribuir a la posibilidad de aplicar ciertas soluciones a otros contextos donde son desarrolladas prácticas de salud para la atención prenatal para adolescentes (25).

\section{CONCLUSIONES}

Los resultados presentados develan buenas prácticas para el cuidado prenatal desde la perspectiva de los profesionales de un centro de referencia para la atención a adolescentes embarazadas. Los hallazgos de este estudio revelan la necesidad de abordar la atención integral a las adolescentes desde la comprensión de las complejidades del embarazo en esta etapa de la vida, lo que requiere de un abordaje multidisciplinar. Así como es recomendable dentro de los servicios de salud sexual y reproductiva el desarrollo de estrategias educativas, el fortalecimiento de la atención en grupos y la inclusión de la red de apoyo de las adolescentes en la atención, para favorecer una experiencia positiva durante el 
cuidado prenatal cuando el proceso de volverse madre acontece durante la adolescencia.

Agradecimientos: agradecemos a los profesionales que amablemente accedieron a participar de esta investigación, al grupo SARHAS por su contribución en la discusión de los resultados de la investigación, y a la Dra.C. Débora Bicudo Faria-Schützer por su rol de co-coordinadora durante la aplicación del grupo focal.

\section{REFERENCIAS}

1. Lloyd CB. Growing up global: the changing transitions to adulthood in developing countries. Washington, D.C., The National Academies Press, 2005.

2. Pinto e Silva JL, Surita FG. Gravidez na adolescência: situação atual. Rev Bras Ginecol Obstet. 2012;34(8):347-50. https://doi.org/10.1590/S010072032012000800001

3. Organización Mundial de la Salud y Organización Panamericana de la Salud. Perfil de los adolescentes y jóvenes de la Región de las Américas. Disponible en: https://www3.paho.org/informe-salud-adolescente-2018/part-one-a-profile-of-adolescents-andyouth-in-the-americas.html

4. Instituto Brasileiro de Geografia e Estatística (IBGE). Projeções da População. Disponible en: https://www. ibge.gov.br/estatisticas/sociais/populacao/9109. projecao-da-populacao.html $=\& \mathrm{t}=$ resultados

5. Organización Mundial de la Salud. El embarazo en la adolescencia. Disponible en: https://www.who.int/es/ news-room/fact-sheets/detail/adolescent-pregnancy

6. Caffe S, Plesons M, Camacho AV, Brumana L, Abdool SN, Huaynoca $\mathrm{S}$, et al. Looking back and moving forward: can we accelerate progress on adolescent pregnancy in the Americas? Reprod Health. 2017;14(1):83. https:/ doi.org/10.1186/s12978-017-0345-y

7. Organización Panamericana de la Salud. Informe final sobre los Objetivos de Desarrollo del Milenio relacionados con la salud en la Región de las Américas. Washington, DC: OPS; 2017:106.

8. World Health Organization. World health statistics 2020: monitoring health for the SDGs, sustainable development goals. Geneva: WHO; 2020:92.
9. Pinto e Silva JL, Surita FG. Pregnancy in AdolescenceA Challenge Beyond Public Health Policies. Rev Bras Ginecol Obstet. 2017;39(2):41-43. https://doi. org/10.1055/s-0037-1600899

10. Flores-Valencia ME, Nava-Chapa G, Arenas-Monreal L. Embarazo en la adolescencia en una región de México: un problema de Salud Pública. Rev. Salud Pública. (Bogotá). 2017;19(3):374-78. https://doi. org/10.15446/rsap.v19n3.43903

11. Holness N. A global perspective on adolescent pregnancy. Int J Nurs Pract. 2015;21(5):677-81. https:// doi.org/10.1111/ijn.12278

12. Córdova Pozo K, Chandra-Mouli V, Decat P, Nelson E, De Meyer S, Jaruseviciene L, et al. Improving adolescent sexual and reproductive health in Latin America: reflections from an International Congress. Reprod Health. 2015;12:11. https://doi.org/10.1186/1742-4755-12-11

13. Pedraza DF, Lins ACL. Complicações clínicas na gravidez: Uma revisão sistemática de estudos com gestantes Brasileiras. Cien Saúde Colet. Disponible en: http://www.cienciaesaudecoletiva.com. br/artigos/complicacoes-clinicas-na-gravidez-umarevisao-sistematica-de-estudos-com-gestantesbrasileiras $/ 17454$ ? id $=17454$

14. Viellas EF et al. Assistência pré-natal no Brasil. Cad. Saúde Pública. 2014; 30 (Suppl. 1): S85-S100. https:// doi.org/10.1590/0102-311X00126013

15. Paim JA. Modelos de atenção e vigilância da saúde. En: Rouquayrol MZ, Almeida FN, organizadores. Epidemiologia e Saúde. $6^{a}$ ed. Rio de Janeiro: MEDSI; 2003. p. 567-86.

16. World Health Organization. WHO recommendations on antenatal care for a positive pregnancy experience. Geneva: WHO; 2016.

17. Ministério da Saúde (BR). Rede Cegonha. Disponible en: https://www.saude.gov.br/saude-para-voce/saudeda-mulher/rede-cegonha

18. Ministério da Saúde. Portaria GM n. ${ }^{\circ}$ 572, de 1 de junho de 2000. Disponible en: http://dtr2001.saude. gov.br/sas/PORTARIAS/PORT2000/GM/GM-572

19. Organização Pan-Americana da Saúde. Padrões globais para serviços de saúde de qualidade para adolescentes. Guia para implementar uma abor-dagem padronizada com o objetivo de melhorar a qualidade dos serviços de 
saúde para adolescentes. Volume 1: Padrões e critérios. Washington, D.C.: OPAS, 2017.

20. Saturno-Hernández PJ. Métodos y herramientas para la realización de ci-clos de mejora de la calidad en servicios de salud. Cuernavaca, México: Instituto Nacional de Salud Pública, 2015.

21. Delgado-Gallego ME, Vázquez-Navarrete ML, MoraesVanderlei L. Cali-dad en los servicios de salud desde los marcos de sentido de diferentes actores sociales en Colombia y Brasil. Rev. salud pública. 2010;12(4):533-45. https://doi.org/10.1590/S0124-00642010000400001

22. Cáceres-Manrique F de M, Ruiz-Rodríguez M, Hernández-Quirama A. La atención prenatal: una mirada desde los profesionales de la salud. Estudio cualitativo. Bucaramanga, 2012. Revista Colombiana de Obstetricia y Gi-necología. Federación Colombiana de Obstetricia y Ginecología; 2015;66(2):103. https://doi.org/10.18597/ $\operatorname{rcog} 12$

23. Tomaz-Moreira SDN, Fagundes-Galvão LLL, MedeirosMelo CO, De Azevedo GD. Fundamentación del abordaje cualitativo para la investigación en salud sexual y reproductiva. Revista Colombiana de Obstetricia y Ginecología; 2007;58(1):28-7. https://doi.org/10.18597/ $\operatorname{rcog} .491$

24. CAISM. Hospital da Mulher Prof. Dr. José Aristodemo Pinotti. Disponible en: https://www.caism.unicamp.br/ index.php

25. Hernández-Sampieri R, Fernández Collado C, Baptista Lucio MP. Metodología de la Investigación. Sexta edición. México: McGraw-Hill, 2014.

26. Bardin L. Análise de conteúdo. Lisboa, Portugal: Edições 70; 2011:118.

27. Campos CJG. Método de análise de conteúdo: ferramenta para a análise de dados qualitativos no campo da saúde. Revista Brasileira de Enfermagem [online]. 2004, v. 57, n. 5, pp. 611-614. https://doi.org/10.1590/ S0034-71672004000500019

28. Salinas Mulder S, Castro Mantilla MD, Fernández Ovando C. Vivencias y relatos sobre el embarazo en adolescentes. Una aproximación a los factores culturales, sociales y emocionales a partir de un estudio en seis países de la región. Panamá: UNICEF Regional Office for Latin America and the Caribbean; 2014.

29. Oliveira PR, Rodrigues JZ, Ferreira JD, Batista DJR, Gusmão RM, Franco SEJ, et al. Gravidez na adolescên- cia: um desafio crítico para os países do cone sul. J. Health NPEPS 2018; 3(2): 506-26. https://doi. org/10.30681/252610103115

30. Vieira EM, Bousquat A, Barros CR, Alves MC. Adolescent pregnancy and transition to adulthood in young users of the SUS. Revista de Saúde Pública. 2017;51(0):25. https://doi.org/10.1590/s1518-8787.2017051006528

31. Chung HW, Kim EM, Lee JE. Comprehensive understanding of risk and protective factors related to adolescent pregnancy in low- and middle-income countries: A systematic review. J Adolesc. 2018; 69:180-88.https:// doi.org/10.1016/j.adolescence.2018.10.007

32. Pastrana-Sámano R, Heredia-Pi IB, Olvera-García M, Ibáñez-Cuevas M, De Castro F, Villalobos Hernán$\operatorname{dez}$ A, et al. Adolescent Friendly Services: quality assessment with simulated users. Revista de Saúde Pública. 2020;54:36. https://doi.org/10.11606/s15188787.2020054001812

33. Ministerio de Salud de Chile. Documento de Sistematización Encuentros de Adolescentes y Salud "En salud los jóvenes eligen”. Santiago de Chile: MINSAL; 2011.

34. Escobal APL, Soares MC, Meincke SMK, et al. Experiences in the process of teenage pregnancy parturition. Rev Fund Care Online. 2016; 8(3):4711-16. https://doi. org/10.9789/2175-5361.2016.v8i3.4711-4716

35. Kane J, Lohan M, Kelly C. Adolescent men's attitudes and decision making in relation to pregnancy and pregnancy outcomes: An integrative review of the literature from 2010 to 2017. Journal of Adolescence. 2019; 72:23-31. https://doi.org/10.1016/j.adolescence.2018.12.008

36. Queiroz MVO, Menezes GMD, Silva TJP, Brasil EGM, Silva RM. Pregnant teenagers' group: contributions to prenatal care. Rev Gaúcha Enferm. 2016;37(esp): e2016-0029. https://doi.org/10.1590/1983-1447.2016. esp.2016-0029

37. Andrade LFB, Rodrigues QP, Silva RCV. Boas Práticas na atenção obstétrica e sua interface com a humanização da assistência. Rev enfermagem de UERJ, Rio de Janeiro. 2017; 25(1): e26442. https://doi.org/10.12957/ reuerj.2017.26442

38. Barbaro MC, Lettiere A, Nakano AMS. Asistencia prenatal a la adolescente y los atributos de la Atención Primaria a la Salud. Rev. Latino-Am. Enfermagem. 2014; 22(1):108-14. https://doi.org/10.1590/01041169.3035 .2390 


\section{FINANCIACIÓN}

El presente trabajo fue realizado con apoyo de la Coordenação de Aperfeiçoamento de Pessoal de Nível Superior - Brasil (CAPES) - Código de Financiamiento 001 y del Programa Latinoamericano para la Investigación en Salud Sexual y Reproductiva (PLISSER).

\section{CONTRIBUCIÓN DE LAS AUTORAS}

Odette del Risco-Sánchez: elaboración del diseño de investigación; trabajo de campo; análisis, interpretación de resultados y elaboración del manuscrito; aprobación de la versión final del artículo.
Erika Zambrano-Tanaka: análisis, interpretación de resultados y elaboración del manuscrito; aprobación de la versión final del artículo.

Natividad Guerrero-Borrego: elaboración del diseño de investigación; análisis, interpretación de resultados y elaboración del manuscrito; aprobación de la versión final del artículo.

Fernanda Surita: elaboración del diseño de investigación; trabajo de campo; análisis, interpretación de resultados y elaboración del manuscrito; aprobación de la versión final del artículo. 